\title{
Research on product styling re-design of household heating equipment with frequency conversion electromagnetism
}

\author{
Su Jianning *, Yin Jinlian, Li Xiaoxiao and Wei Jin \\ School of Design Art, Lanzhou University of Technology, Lanzhou, Gansu, 730050, China
}

\begin{abstract}
In view of the homogeneity and lack of characteristics in the form design of the heating equipment with frequency conversion electromagnetism, the research was carried out with the re-design method. Firstly, the emotion requirements of users, enterprises and market were investigated, and the product styling re-design was positioned; Then according to the target position, the styling of the representative products was selected to be analyzed, and the key design elements were extracted; Finally, according to the practical situation, the key design elements were integrated and innovated, and a kind of new product form was re-designed subsequently. The research shows that the scheme selected by the enterprise meets the actual demand, and the re-design method can effectively improve the quality of product form.
\end{abstract}

\section{Introduction}

In the past the coal-fired boiler was widely used for heating in the North China, and the emission of dust and harmful gas such as carbon monoxide and sulfur dioxide made the air pollution situation get worse. In the South China, the air-conditioner is mostly used for heating, but its shortcomings are also obvious. For instance, the inside of the air-conditioner is hard to clean, which leads ash accumulated to make people more likely to suffer from air-conditioner disease ${ }^{[1]}$. Therefore, electric heating has gradually become the main option. The traditional electric heating equipment mainly uses the resistance radiation or electromagnetic induction, which has the problems of low efficiency and short service life. Heating equipment with frequency conversion electromagnetism adopts frequency conversion electromagnetic induction technology to produce heat, which is more efficient, more energy efficient and environmental-friendly. Hence it will become the first choice for the enterprises when developing new products.
After the completing of product function, some enterprises pay attention to the re-design of product form when upgrading their products.

A good product styling is the coordination of "form" and "perception". "Form" means the appearance of the product which refers to its material objective expression. "Perception" means the image or expression embodied by the product "form", which refers to the mood or feelings expressed by the product. In the process of users' cognition, the "form" of design reflects the "perception". Therefore, in the process of innovative design, the "form" of design should be determined by the need of "perception". These two are interdependent and combine a complete whole. Ignoring styling design will lead to product homogeneity and lack of characteristics ${ }^{[2]}$, and the product quality can not express well.

Based on what discussed above, for the heating equipment with frequency conversion electromagnetism of the project entrusting company, the re-design theory and method were applied to carry out innovative design for its product styling, and the specific process is shown in Figure 1.

*Corresponding author's e-mail: sujn@lut.cn 


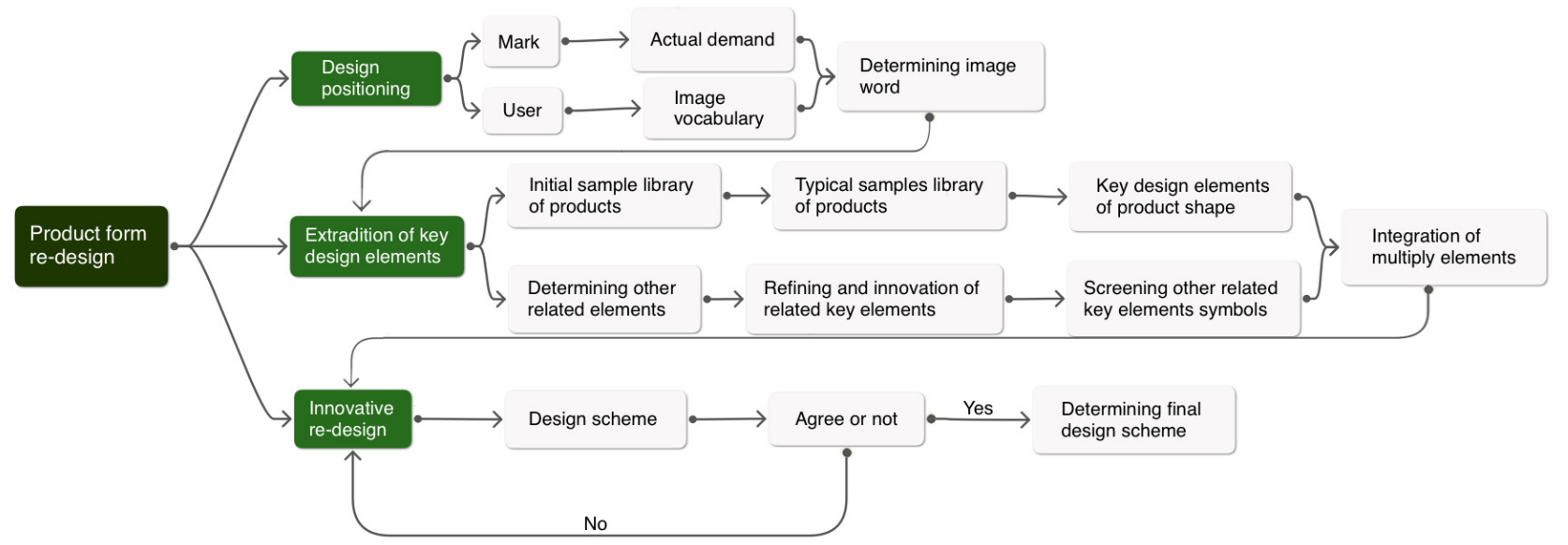

Figure 1 product styling re-design process

\section{Concept of the re-design}

Since the second half of 2015, PERA Global released the industrial re-design strategy ${ }^{[3]}$ in order to promote the implementation of the "Made in China 2025" strategy and solved the major obstacles and problems during the transformation and upgrading of China's manufacturing industry. "Re-design" is to reinterpret the original design from a contemporary perspective ${ }^{[4-5]}$. The reinterpretation can be nostalgic, imitative, simplified or ordered ${ }^{[6]}$. Kenya Hara first put forward this concept ${ }^{[7]}$. Its core idea is to defamiliarization of things familiar to us, to return to the origin and to redefine the value of the products. It is a new kind of design thought and method. On the basis of the original design combined with its own characteristics, conducting the Meta re-design makes the products more reasonable and more suitable for their own development, which effectively improves product value.

\section{The product styling re-design positioning}

The styling of products will change with the function of products, the needs of users and the development of the times, which is the objective law of evolution of the product styling. The accuracy of design positioning will directly affect the success of product design and development ${ }^{[8-9]}$. In the early stage of the product styling re-design, the designers need to make clear the direction of design through the detailed analysis of social needs and the product features.

\subsection{Market survey}

With the increase of the national economy and the rapid development of science, technology and culture, the market demand for heating equipment will continue to grow, and the environmental problems caused by heating can not be ignored. The heating equipment with frequency conversion electromagnetism has a broad market and great potential. Through the market survey of heating equipment, it is found that the market demand for heating equipment is increasingly abundant and diversified. The consumption upgrade and economic transition make many consumers and dealers begin to pay attention to the product styling and function design. The heating equipment industry is developing towards the direction of functional diversification, intelligence and product styling aesthetics.

\subsection{User research}

User's demand is the main reason to promote product re-design. User's demand ${ }^{[10]}$ is divided into functional demand and emotional demand. Functional demand is the rational demand of users, which is the basis of product re-design, while emotion demand refers to the kansei demand of users, which is the guide of product re-design. The main factor for the success of product design is determined by the needs and preferences of users $^{[11]}$.

According to the users of different occupations, the interview survey was carried out. The experience of different users using heating equipment was sorted out, analyzed and studied. In terms of the heating equipment with frequency conversion electromagnetism, the user not only need to consider its functional requirements, but also had a certain preference for its product styling image. Through the market visits and user surveys in the early stage, the vocabulary of the image preference of the heating equipment with frequency conversion electromagnetism was preliminarily screened, as shown in Table 1.

Table 1 Product perceptual image vocabulary

\begin{tabular}{|c|c|c|c|c|}
\hline Concise & Steady & Ponderable & Mechanical & Precise \\
\hline Safe & Multi-functional & Technological & Easy-to-use & Interactive \\
\hline
\end{tabular}




\begin{tabular}{|c|c|c|c|c|}
\hline Adaptive & Exquisite & Artistic & Cozy & Operational \\
\hline Material & Beautiful & Reliable & Complex & Strength \\
\hline
\end{tabular}

\subsection{Survey summary}

An expert group was formed which contains the design team, enterprise management and sales personnel to conduct the secondary screening of the product image vocabulary and obtain three target kansei images: "safe", "beautiful" and "mechanical". At the same time, the representative of the enterprise proposed that the product styling should embody certain cultural connotation, so as to achieve the purpose of strengthening the cultural connotation of the enterprise and improving the product identification. Therefore, the target kansei image of "cultural" was added into the re-design of the household heating equipment with frequency conversion electromagnetism.

\section{Extraction of the product styling re-design elements}

\subsection{Selection of the product prototype}

Product styling directly affects the user's sensory impression. It is an important factor to the success of product re-design ${ }^{[12]}$. Different products have different function characteristics and styling characteristics. Its function characteristics determine the styling characteristics, and styling characteristics reflect the function characteristics. In the initial stage of product styling re-design, it is necessary to have a deep understanding of the existing products and the same type products to analyze the prototype of products and to identify their styling characteristics.

For the heating equipment with frequency conversion electromagnetism, its function is different from other products in the market. As the winter heating equipment in some areas, its styling characteristics must meet the demand for heating function. By visiting the shopping malls, sales areas and enterprises, according to the three objective kansei image of "safe", "beautiful" and "mechanical", the domestic and foreign heating equipment pictures were collected from various channels, such as the product brochure of heating equipment, similar products in the market and the internet. Because the similar products generally lacked the image of "cultural", there were not enough products with "cultural" image for selecting. In that case, the "cultural" image was not considered. After preliminary screening, a sample library was established. For convenience, the sample pictures were amended for selecting. The samples picture angle was inclined (left squint/right squint) to show as many sample details as possible.

\subsection{Analysis of the product samples}

Through the analysis of the initial sample library of products, it was found that the products of various brands of heating equipment were similar in styling design. The product styling was mostly cubic, big, straight and neat. Occasionally the curve and broken line was used as change, but on the whole the styling was indifferent and cubic which looked clumsy, plan and gave a sense of rationality. The product design of the samples above mostly only reflected the functionality of the product, and the styling design lacked of innovation and the cultural connotation.

According to the analysis results of the initial samples, five representative samples with the target kansei image were selected after discussion by the experts. After the styling design symbols of representative samples were analyzed, the results are shown in Table 2.

Table 2 Styling design symbol of representative samples analysis

\begin{tabular}{|c|c|c|c|}
\hline \multicolumn{2}{|c|}{ Representative sample } & \multicolumn{2}{|r|}{ Styling symbols } \\
\hline \multirow{4}{*}{ Y1 } & \multirow{4}{*}{$1_{1}^{00}$} & Overall profile A & Combination of straight line and arc \\
\hline & & display screen B & Straight type \\
\hline & & Color $\mathrm{C}$ & Manly white, supplemented by blue \\
\hline & & Ventilation hole $\mathrm{D}$ & Round type distributed upper right \\
\hline \multirow{4}{*}{ Y2 } & \multirow{4}{*}{ restal } & Overall profile A & Combination of straight line and polyline \\
\hline & & Display screen B & Tilting \\
\hline & & Color C & Mainly white, supplemented by orange \\
\hline & & Ventilation hole D & Small dots type surrounding the body \\
\hline \multirow{4}{*}{ Y3 } & \multirow{4}{*}{$E "$} & Overall profile A & Combination of straight line and polyline \\
\hline & & Display screen B & Tilting \\
\hline & & Color C & Mainly white and orange \\
\hline & & Ventilation hole D & None \\
\hline \multirow{2}{*}{ Y4 } & \multirow[t]{2}{*}{ 田 } & Overall profile A & Combination of straight line and polyline \\
\hline & & Display screen B & Straight type \\
\hline
\end{tabular}




\begin{tabular}{|c|c|c|c|}
\hline \multirow{2}{*}{} & & Color C & Mainly white and red \\
\cline { 3 - 4 } & & Ventilation hole D & None \\
\hline \multirow{4}{*}{ Y5 } & \multirow{4}{*}{} & Overall profile A & Combination of straight line and curve \\
\cline { 3 - 4 } & \multirow{3}{*}{} & Display screen B & Straight type \\
\cline { 3 - 4 } & & Color C & Mainly silver gray \\
\cline { 3 - 4 } & & Ventilation hole D & None \\
\hline
\end{tabular}

\subsection{Extraction of the product styling design symbols}

symbols were summarized and refined. The results are shown in Table 3. After simplifying or deforming the design symbols, it can guide the subsequent product styling re-design.

The representative product samples were analyzed to obtain the styling design symbols and the styling design

Table 3 Extraction of design styling symbols

\begin{tabular}{|c|c|}
\hline \multirow{2}{*}{ Item } & Styling symbols \\
\hline \multirow{2}{*}{ Overall profile A } & Combination of straight line and curve \\
\cline { 2 - 2 } & Combination of straight line and polyline \\
\hline \multirow{2}{*}{ Color B } & Mainly white supplemented by other color \\
\cline { 2 - 2 } Display screen C & Other color \\
\cline { 2 - 2 } & Straight type \\
\hline \multirow{2}{*}{ Ventilation hole D } & Tilting \\
\cline { 2 - 2 } & Upper right side \\
\hline
\end{tabular}

The entrusting company is located in Lintao County,

\subsection{Extraction of the cultural image element}

The product culture refers to the integration of representative landscape patterns, customs and culture or historical signs in the product, so that the products have unique cultural characteristics. Cultural symbols are the symbols with representative meanings and carriers of cultural information. The symbols can not only refer to things, but also express emotions and concepts ${ }^{[13]}$. The cultural symbols are the precious wealth of the human beings. Integrating cultural consciousness into the product re-design ${ }^{[14]}$ is a very useful design resource in the process of product styling re-design, which can enable the re-designed products to reflect certain cultural connotations on the basis of function.

Table 4 Symbol extraction of cultural image

\begin{tabular}{|c|c|c|}
\hline Lintao & Extraction of representative item & Image form symbol transformation \\
\hline \multicolumn{3}{|l|}{$\begin{array}{l}\text { Representative } \\
\text { item }\end{array}$} \\
\hline & & Simplification \\
\hline \multicolumn{3}{|l|}{$\begin{array}{l}\text { Representative } \\
\text { decorative pattern }\end{array}$} \\
\hline & & Deformation \\
\hline
\end{tabular}




\section{Products styling innovative re-design}

Through the market visits, user surveys and the reconstruction of similar products in the market, the product styling re-design should better reflect the target image needed by the users, enterprises and market. On the basis of the original products, combining the features of the product function, the Meta re-design was carried out to make the product styling better meet the needs, and improve the identification of the product itself.

Based on the analysis above and the functional characteristics of the heating equipment with frequency conversion electromagnetism, the extracted styling design symbols were integrated and innovated. At the same time, according to the modality aesthetic law, the Meta re-design was carried out for the four target images of "safe", "mechanical", "beautiful" and "cultural", and a variety of product styling re-design schemes were obtained. After evaluation and screening by the experts of the enterprise, the best design scheme was determined

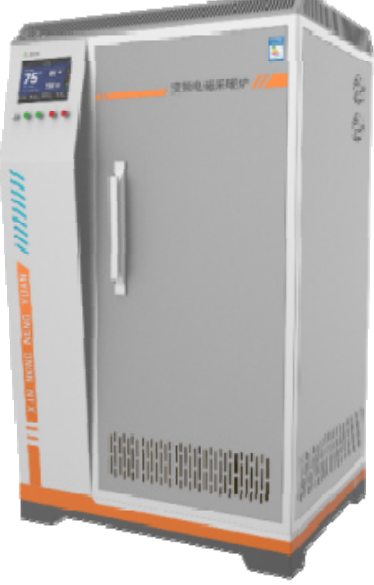

and shown in Figure 2. On the basis of absorbing and extracting the design symbols reasonably, the integration and innovation was conducted for the scheme effectively. As shown in Table.4, the ventilation hole was a reasonable deformation of regional cultural symbols decided by "cultural" image. Meanwhile, the design of the ventilation hole was decided by the function and operation safety, and this scheme was a coordination result between the "safe" and "cultural" image of styling design and the equipment function. Table 3 made it explicit that both two kinds of combination can deliver the image of "mechanical". Integrating the enterprise expert opinion and extracted styling design symbols, the combination of broken lines and straight lines was used to highlight the image of "mechanical". Also the scheme of white with other colors as supplement was chosen as the color scheme, in order to meet the "aesthetic" needs of the users and market. At same time considering the ergonomics rationality, the display screen was tilting so that it can be operated more easily.

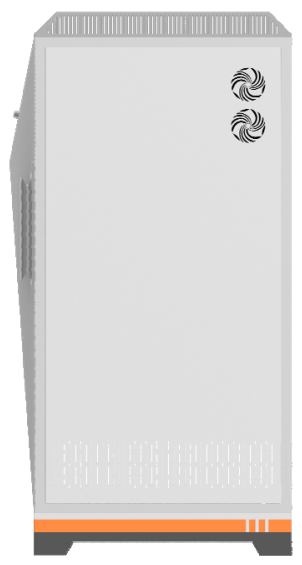

Figure 2 The best design scheme

Generally speaking, the re-designed new product styling can clearly convey the four design target position images of "safe", "mechanical", "beautiful" and "cultural", which makes the product styling not only have the product function characteristics, but also enhance the enterprise identification of the product. It avoids the homogenization of the same type of product in the market, and meets the emotional needs and demands for cultural connotation from the users, market and enterprises.

\section{Conclusion}

Product styling re-design is to reshape the product styling according to the user's demanded "perception", so as to achieve the coordination and unity of "form" and "perception". In this research, based on the heating equipment with frequency conversion electromagnetism of the project entrusting company, starting with the design positioning, the key design elements and cultural symbols were extracted and the innovation design was conducted for the products styling to get a highly recognized re-design scheme by the entrusting company.
Product styling re-design can not only improve the product quality, but also improve the product brand recognition. Enterprises should firmly grasp the pulse of the times, keep pace with the times, timely use the theory of re-design method, transform the new user needs into design elements, create the excellent products that meet the development of the times and user needs, and actively enhance the competitiveness of the enterprises.

\section{References:}

1. Hu Yuan. (2014) Design and Research for Electric Heater. South China University of Technology.

2. Lv Yan. (2014) Product design theory and method of "redesign" the research. Kunming University of Science and Technology.

3. Tian Feng. (2015) Industrial re-design break through "made in China 2025". Journal of intelligent manufacturing, (10): 32-34.

4. Celik H K, Mihaela E. Lupeanu. (2013) Product re-design using advanced engineering applications and function analysis: a case study for greenhouse clips.Journal of the Brazilian Society of Mechanical 
Sciences \& Engineering, 35(03):305-318.

5. Smith S, Smith G, Shen Y T. (2012) Redesign for product innovation. Design Studies, 33 (2):160-184.

6. He Fang. (2018) Research on Chinese traditional graphic redesign method. Art 100, 34 (03): 185-191

7. Kenya Hara, Ji Jiang Hong. (2010) Designing design. Guangxi Normal University Press, Guilin.

8. Hu Junhong. (2001) Product design positioning concept. Packaging Engineering, 22(06):77-78.

9. Pina P, Guilherme Góis, Fábio Mano, et al. (2017), .A sentiment analysis solution for the re-design of product-service systems. International Conference on Engineering. Madeira Island, Portugal.

10. Du hemin. (2018) Research on product form design based on user demand. Packaging Engineering, 39 (04): 104-107

11. Edwards K L. (1995) Product design: Practical methods for the systematic development of new products. Materials and Design, 16(5):301-301.

12. Li xiangwen. (2016) On the strategy and method of automobile modelling design under the background of replacement. Journal of Wuhan University of Technology (Social Science Edition), 29(02):308-313.

13. Zhu Shangshang, Luo Shijian, Ying Fangtian et al. (2010) Product family design DNA supporting product visual recognition. Journal of Zhejiang University (Engineering Edition), 44 (04): 715-721

14. Lu Keqi, Pan Rong. (2016) Product redesign based on Chinese spirit. Packaging Engineering, 37(18):121-124.

15. Wang Xu. (2019) Design of simple intelligent water dispenser based on morphological analysis. Machine Manufacturing and Automation, 48 (03): 140-142 